\title{
The link between CMEs, filaments and filament channels
}

\author{
S. F. Martin ${ }^{1}$, O. Panasenco ${ }^{1}$, O. Engvold ${ }^{2}$, and Y. Lin $^{2}$ \\ ${ }^{1}$ Helio Research, 5212 Maryland Avenue, La Crescenta, CA, 91214, USA \\ ${ }^{2}$ Institute for Theoretical Astrophysics, University of Oslo, Oslo, Norway
}

Received: 17 October 2007 - Revised: 17 December 2007 - Accepted: 15 January 2008 - Published: 15 October 2008

\begin{abstract}
We present a broad concept for the build-up to eruptive solar events which needs to be tested in future observational and theoretical research. In this concept an eruptive solar event consists of a coronal mass ejection, a filament eruption, a cavity around the filament, and a flare. In our picture, the initial energy source must be external to this eruptive system but also feed into it. Among all eruptive events the common denominator is a filament channel with canceling magnetic fields along a primary polarity reversal boundary. We find that magnetic reconnection at or close to the photosphere is the only interpretation of canceling fields to date that is consistent with observations of filament channels. This reconnection serves to transfer magnetic flux from the photosphere into the chromosphere and corona along polarity reversal boundaries and concurrently initiates the building of a filament channel. Magnetic flux, in excess of that needed to sustain the filament channel, goes into building a filament magnetic field that is always aligned with the polarity reversal boundary and the channel magnetic field. The filament magnetic field remains separated from overarching coronal magnetic fields by the magnetic field of the cavity. The magnetic flux being transported upward from the photosphere/chromosphere carries streams of plasma into the corona along the filament magnetic field. However, the flowing and counterstreaming filament mass also slowly drains out of the field and thereby leaves behind new strands of cavity magnetic field with little or no associated mass. When the build-up of magnetic pressure in the filament and cavity magnetic fields exceeds that of the overlying coronal loops, the coronal loops, the filament and the cavity together begin an observable slow rise which can last a few hours to many days before rapid onset and ejection with a solar flare. We suggest that this process can be accelerated by any number of external triggering mechanisms which serve as catalysts
\end{abstract}

Correspondence to: S. F. Martin

(sara@helioresearch.org) to cause the impending eruption to happen earlier than it otherwise would occur.

Keywords. Solar physics, astrophysics, and astronomy (Magnetic fields; Photosphere and chromosphere)

\section{Introduction}

The objective in this paper is to present the hypothesis that CMEs are causally linked to the formation, evolution and maintenance of filament channels, filaments, and filament cavity filament magnetic fields. We proceed step by step to present the rationales for how and why CMEs can be linked back to their ultimate energy source in dynamics associated with canceling magnetic fields in the photosphere beneath eruptive events.

Cancellation of magnetic fields is a graphic observational term defined as "the disappearance of magnetic flux at the mutual boundary of opposite polarity magnetic fields" (Martin et al., 1985). The observed canceling magnetic fields can be interpreted in several different ways (Zwaan, 1985). We illustrate how canceling magnetic fields can represent the transfer of line-of-sight fields in the photosphere to transverse fields in the corona. Observations, however, have been lacking to fully observe and understand this transformation that is fundamental to the long, slow build-up to eruptive events that include the components: CMEs, erupting filaments, erupting cavities, and flares. Surges, flare waves, and other observed phenomena accompanying eruptive flares are regarded as secondary associated events rather than primary components of eruptive solar events.

\section{Essential definitions, clarifications and associations}

The term "eruptive solar flares" in the title of the IAU 133 Colloquium book on "Eruptive Solar Flares" (Svestka et al.,

Published by Copernicus Publications on behalf of the European Geosciences Union. 


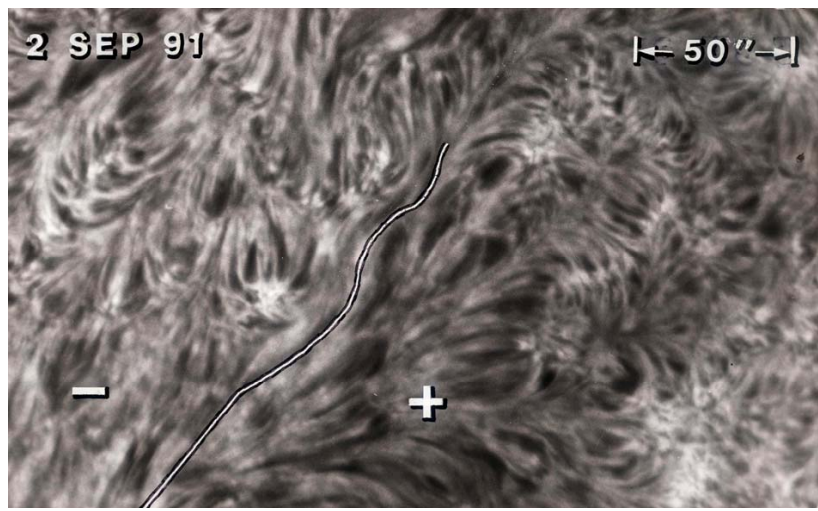

Fig. 1. An empty filament channel is recognized in an $\mathrm{H} \alpha$ image from the Big Bear Solar Observatory. A line is drawn along the division between the opposite polarity network fields. The fibrils are parallel with the polarity boundary and do not cross it. On each side, and relative to the plagettes, the chromospheric fibrils have an apparent root end and a more pointed end. On the positive network side, the apparent root end is in the positive polarity fields and the fibrils point toward negative magnetic fields. On the negative network side of the filament, the negative polarity is at the apparent rooted end and the fibrils point towards positive polarity fields. This pattern reveals that the magnetic fields have the same directions on the two sides of the filament channel and this allows us to deduce the sense of rotation of the field in a cross-section of the filament channel perpendicular to the polarity reversal boundary.

1992) was intended to distinguish events that include a coronal mass ejection (CME), erupting filament, erupting cavity and flare from simple "confined flares" that are not associated with this combination of associated events. The term "CME" has been used by some authors to refer to the whole physical system including the CME (coronal front of an event), the erupting filament, erupting cavity and the flare (review by Forbes, 2000) although CME originally meant only the coronal event originating from coronal loops without associated events. Regardless of the choice of definitions, there has been recognition, for over a decade, of close associations between CMEs, erupting filaments, filament cavities and flares. For the purpose of presenting our concept we will assume that the associations between these events is nearly 100 percent. Then it is easier to recognize that all of these events are parts of a single magnetic system that can erupt. If this assumption is true, we must look outside of this system to find the true cause of eruption of the whole system.

\section{Common denominators among CMEs, flares and erupting filaments}

One question we should ask is: what have CMEs, flares and erupting filaments in common? The answer is known. All of these events, individually and as a system, are located above and around places where the magnetic field changes polar- ity, places known as a "neutral line", a "polarity inversion" or a "polarity reversal boundary". We know that erupting filaments, in particular, originate from specific polarity reversal boundaries that are also within filament channels (Martin, 1990; Gaizauskas, 1998). We also already know they only come from filament channels that have reached maximum development; i.e. have reached maximum magnetic shear along the polarity boundary (Martin, 1998). So we should next address the questions: What are filament channels and how do we recognize when one has reach maximum development?

\section{Filament channels}

A filament channel can be loosely defined as the volume of space in which a filament forms and resides and which is identified by specific features of the photosphere, the chromosphere and low corona. Completely developed filament channels are characterized by three properties itemized below and also illustrated in Fig. 1.

1) The formation of specific filament channels in the chromosphere have been described by Smith (1968), Martin (1990), Gaizauskas et al. (1997), and Wang and Muglach (2007). In the chromosphere a filament channel consists of fibrils aligned along a polarity reversal boundary. This is the equivalent of saying that these are locations where the vector magnetic field is aligned with the fibrils and the polarity boundary. In the photosphere, vector magnetograms have shown that the magnetic field in the photosphere is in the same direction as fibrils in the chromosphere within the accuracy of the resolution of the magnetograms which, in most previous observations, has been less than the resolution of $\mathrm{H} \alpha$ images (Martin, 1990, 1998).

2) In the low corona, small loops parallel with the polarity reversal boundary, and not crossing it, are the evidence that the channel in the photosphere and chromosphere also extends into the low corona (Wood and Martens, 2003).

3) No fibril crosses the polarity reversal boundary. This is the equivalent of saying that no magnetic field lines from active region or network magnetic fields cross the polarity boundary in the magnetic shear zone around the polarity reversal boundary, in the chromosphere above, or within filaments.

A smoothed schematic representation of the general magnetic field of a filament channel is given in Fig. 3 of Martin et al. (1994). As deduced from chromospheric filbrils, the magnetic field direction smoothly changes from upward on one side of a polarity reversal boundary to horizontal along the polarity reversal boundary and to vertically downward on the other side of the polarity boundary.

Filament channels are apparently relatively stable against magnetic reconnection above the photosphere as there are no oppositely directed magnetic fields that come into contact before an eruptive event except at the photosphere where 
flows drive the photospheric footpoints of opposite polarity fields together. The zone of horizontal field in the middle of the channel exists in the photosphere, in the chromosphere (Fig. 1) and in the corona to the sides of filaments. In active regions, the zone of horizontal field extends vertically from the photospheric polarity boundary to at least the top of the filament spine. For the most quiescent filaments, the horizontal zone only exists where opposite polarities meet. It does not necessarily include the space between the network cell boundaries. The significance of filament channels is that their magnetic fields deviate substantially from a potential field configuration. Therefore, filament channel magnetic fields represent locations on the Sun where energy is being stored continuously.

The evolution of filament channels shows evidence of their accumulating energy in the photosphere, chromosphere and low corona. During a CME the photospheric and chromospheric part of the channel reveal little evidence of changing configuration. Hence, these layers of the filament channel apparently do not give up much energy during a CME. Therefore, we deduce that the coronal part of the channel is the primary site where energy is stored and later released during eruptive solar events. The coronal part of the channel includes not only the magnetic field and plasma associated with the filament and filament cavity magnetic field, but also low coronal loops next to and parallel with filaments as seen in $171 \AA$, and $195 \AA$ images from the EIT experiment on SOHO (Wood and Martens, 2003).

The big unanswered question is: how do the coronal parts of the filament channel store energy? We look to the magnetic fields in the bottom of the filament channel to see what filament channels have in common. We find that all filament channels with filaments also are sites where magnetic fields are canceling.

\section{Canceling magnetic fields}

Litvinenko (1999), Litvinenko and Martin (1999), Litvinenko and Wheatland (2005), Litivenko et al.. (2007) reason that magnetic reconnection near the photosphere accounts for canceling magnetic fields as well as the concurrent formation of filament magnetic fields. Also according to several filament formation theories, magnetic reconnection associated with canceling magnetic fields, converts short field lines into longer field lines (Bogdan and Rompolt, 1986; van Ballegooijen and Martens, 1989; Martens and Zwaan, 2001). The interpretation of reconnection at or near the photosphere is consistent with a number of theoretical and observational considerations. First, reconnection is most likely to occur at the temperature minimum (Litvinenko, 1999). Secondly, along filament channels, there is an absence of conspicuous brightening; this is consistent with reconnection taking place in a region of high density where the reconnection proceeds very slowly and inconsistent with occurrence in low density regions such as the chromosphere or corona where known types of reconnection proceed very rapidly. Third, the configuration of the magnetic field in the chromospheric and coronal parts of filament channels is not favorable for reconnection because the magnetic field rotates smoothly, being upward on one side of the channel and downward on the other side. Hence the magnetic field of a channel is more resistant to reconnection in the chromosphere and corona than in the photophere where the field is driven by flows.

Reconnection at the photosphere can be slow, relatively steady, and without visible effects. Reconnection in the chromosphere or corona is more intermittent and energetic with conspicuous flaring as a result of particle acceleration and heating. Because of these differences, cancelation is not thought by us to be the direct cause of flares. Rather, canceling fields build filament channels and filament channels happen to be an environment in which flares can occur under certain conditions.

Other interpretations of the apparent disappearance of magnetic flux were discussed by Zwaan (1985). One was simple submergence. Another was upward retraction of $U$ loops. Yet another was magnetic reconnection above the photosphere. None of these interpretations is consistent with our data on filament channels. It is important that the observations show that (1) the motion of the canceling fields are perpendicular to the direction of the polarity reversal boundary and that (2) the local field direction is nearly parallel to the local polarity reversal boundary. This is consistent with a lack of prior connection beneath the photosphere and hence both options of simple submergence and emerging $U$ loops are unlikely. Both require the prior existence of magnetic field joining opposite polarities; these would necessarily cut across the magnetic fields of the fibrils in the filament channel. To our knowledge no evidence of such field or structure, cutting across the polarity reversal boundary in a filament channel, has been observed in low or high quality observations acquired to date, except during small or large flares.

The interpretation of magnetic reconnection above the photosphere also does not adequately explain canceling magnetic fields. Evidence of such reconnection does occur frequently during solar flares of all sizes. However, observations clearly show that flare loops do not submerge; successive loops increase in height. Additionally, the time scale of reconnection above the photosphere is too fast. Thus, of the possibilities suggested and depicted by Zwaan (1985), only magnetic reconnection at or very close to the photosphere is consistent with the overall magnetic configuration of filament channels.

Consistent with the above references, in our view, canceling magnetic fields are the single visible manifestation of a process by which line-of-sight magnetic flux is extracted from the photosphere and stored first in the fibril fields of the chromosphere and the extension of some of these fibril fields into the corona. When the condition of maximum magnetic shear is reached in a filament channel, the magnetic flux 


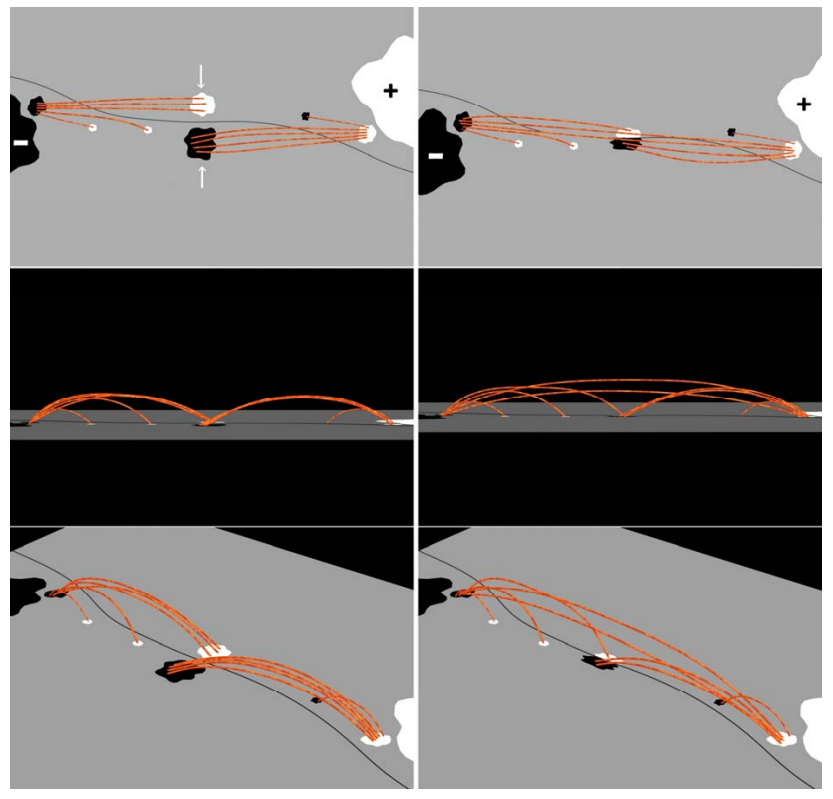

Fig. 2. A thread model schematic of how we interpret the observed canceling magnetic fields. White represents positive polarity and black is negative polarity. Cancellation is interpreted as magnetic reconnection near the photosphere in which the line-of-sight magnetic field in the photosphere is transferred to horizontal magnetic field in the corona. The filament spine forms along the resulting horizontal component of the field in the low corona and between the more vertical opposite polarity magnetic fields.

from the canceling magnetic fields is converted to primarily transverse fields in the low corona along the polarity reversal boundaries. The process is illustrated in Fig. 2. At this stage the fields are necessarily pushed together sideways by photospheric motions after a filament channel has formed. This illustrates why filaments lie along polarity reversal boundaries; filaments are dependent on and confined by the magnetic field geometry of the filament channel.

This photospheric reconnection process is also capable of lifting mass into the low corona (Litvinenko et al., 2007). The mass does not necessarily stay in the corona; it merely flows along the magnetic field (Lin, 2002, 2004; Lin et al., $2003,2005,2008)$. The flows are bi-directional and are interleaved on a small scale. The collective process of flows is known as "counterstreaming" (Zirker et al., 1998; Lin et al., 2003). There is no evidence, in the cited high resolution studies, of deceleration or reversal of direction of mass flows along filament threads. Hence, in quiescent conditions, reversals in the direction of mass flows are not taking place in the coronal parts of filaments. It is not known whether the flows reverse direction after flowing into the chromosphere/photsphere interfaces or whether all flows are newly initiated concurrently with each of many tiny reconnections at the photosphere. If all flows are newly initiated along magnetic threads, newly elevated from the photosphere into the

\section{STAGES IN THE DEVELOPMENT OF A CME}

\begin{tabular}{|c|}
\hline CME + Erupting Filament + Cavity + Flare \\
\hline Expanding Coronal Loop System \\
\hline Increasing Cavity Magnetic Field \\
\hline Filament Magnetic Field Formation \\
Filament Channel Formation \\
Canceling Magnetic Flux \\
\hline Solar Convection + Active Region Evolution \\
\hline
\end{tabular}

Fig. 3. Diagram of observed and inferred stages in the build-up to eruptive events beginning with the lowest block and proceeding in approximate sequential order to the eruptive event represented at the top. The process can continue through a series of eruptive events until the canceling flux ceases or falls below an assumed critical level.

corona, then we conclude that there must be many older filament threads along which mass is no longer flowing. In our picture there is a relationship, yet to be quantified, between the amount of magnetic field that is cancelled and the amount of magnetic flux stored in the chromosphere and corona.

This magnetic field built up in the corona during the lifetime of filaments (Lin et al., 2005), in our view is mostly the magnetic field that has canceled during the lifetime of a filament and possibly during the development of the filament channel. Eventually, it is inevitable that the magnetic field of the filament and cavity would rise (Filippov et al., 2005) and come into balance with the overlying coronal arcade field. Thereafter, the filament and cavity magnetic field exert continuous outward pressure on the overlying coronal arcade. The coronal arcade then begins a very slow rise that can last at least four days (Hundhausen, 1999) and similarly for the filament below (Joshi and Srivastava, 2007; Martin et al., 2006). Eventually the coronal arcade can no longer suppress the filament and cavity magnetic field and the rising magnetic system then begins to accelerate until the more cataclysmic reconnection of a solar flare further boosts the outward acceleration of the rising system. The filament, cavity and overlying corona act as a single system rising and erupting together. One does not cause the other nor is the eruption caused by the flare. Rather all are a consequence of the long slow build-up of magnetic flux being fed into the coronal magnetic system from the filament channel. 


\section{The triggering of eruptive events}

Much attention has been given to the triggering of eruptive events. Common mechanisms proposed as the possible triggers for flares have been interactions with emerging magnetic flux (Rust, 1976; Wang and Sheeley, 1999; Feynman and Martin, 2001; Xu et al., 2007), interactions of filament magnetic fields with overlying coronal fields (Antiochos et al., 1999), interactions of the root fields of filaments with adjacent magnetic fields, the occurence of small solar flares (Nagashima et al., 2007), and in relatively rare cases, being hit by flare waves (Harvey et al., 1974; Okamoto et al., 2004). The concept presented here is not dependent upon any particular triggering mechanism. Any number of such interactions could serve as a catalyst to cause an impending event to erupt earlier than it would if the catalyst were not present. It is not the purpose of this paper to make any judgement as to what final trigger(s) of eruptive events might occur. Rather we choose to recognize that any number of triggering mechanisms are possible and are important to understanding exactly when any given eruptive event might occur. We concentrate on fundamental processes which should result in eruptive events independent of any final triggering mechanisms that come into play.

\section{Concluding remarks}

We have presented the various stages of our concept of how CMEs are linked to the evolution of filament channel and filament magnetic fields and have summarized these stages in Fig. 3. We assume that the parts of this concept will be tested in future observational and theoretical research. Principal among the many questions that need to be addressed are: (1) Is there a quantitative relationship between the amount of canceling magnetic flux observed in a specific filament channel during the lifetime of a filament, the energy in this magnetic flux, and the energy needed to cause the overlying corona to expand at the observed rate? (2) Can photospheric magnetic reconnection be simulated quantitatively and realistically? (3) In high resolution observations, can the initial input of mass into filaments and the draining of mass out of filaments be observed? (4) Can the input of small knots of mass into filament threads be associated with the rate and regularity of canceling magnetic fields?

Acknowledgements. S. F. Martin and O. Panasenco were supported by NSF grant ATM-0519249. Y. Lin acknowledges the Norwegian Research Council grant FRINAT171012. The authors have benefitted by stimulating discussions with V. Gaizauskas, J. Zirker, T. Forbes, Y. Litvinenko, D. Mackay, N. Srivastava, A. Pevtsov and K. S. Balasubramaniam.

Topical Editor R. Forsyth thanks two anonymous referees for their help in evaluating this paper.

\section{References}

Antiochos, S. K., DeVore, C. R., and Klimchuk, J. A.: The Structure of Solar Prominences, Astrophys. J., 510, 485-493, 1999.

Forbes, T. G.: A Review on the Genesis of coronal Mass Ejections, J. Geophys. Res., 105(A10), 23 153-23 165, 2000.

Feynman, J. and Martin, S. F.: The Initiation of Coronal Mass Ejections by Newly Emerging Magnetic Flux, J. Geophys. Res., 100, 3355-3367, 1995.

Filippov, B. P., Den, O. G., and Zagnetko, A. M.: Coronal and Stellar Mass Ejections, IAU Symposium Proceedings of the IAU 226, Held 13-17 September, Beijing, edited by: Dere, K., Wang, J., and Yan, Y., Cambridge: Cambridge University Press, pp. 464-469, 2005.

Gaizauskas, V.: Essential Ingredients for Filament Formation (Review), in: New Perspective on Solar Prominences, IAU Colloquium 167, ASP Conf. Series, 150, 257-264, 1998.

Gaizauskas, V., Zirker, J. B., Sweetland, C., and Kovacs, A.: Formation of a Solar Filament Channel, ApJ, 479, 448, 1997.

Harvey, K. L., Martin, S. F., and Riddle, A. C.: Correlation of a Flare-Wave and Type II Burst, Sol. Phys., 36, 151-155, 1974.

Hundhausen, A.: Coronal Mass Ejections, in: The many faces of the sun: a summary of the results from NASA's Solar Maximum Mission, edited by: Strong, K. T., Saba, J. L. R., Haisch, B. M., and Schmelz, J. T., New York: Springer, p. 143, 1999.

Joshi, V. and Srivastava, N.: On the study of kinematics of eruptive quiescent prominences observed in He $304 \AA$, Proc.of The 2nd UN/NASA IHY Workshop on the International Heliophysical Year and Basic Space Science, Bangalore, B. Astron. Soc. India, 35, 447-455, 2007.

Lin, Y.: Oscillation and Flows in Sub-structure of a Large Filament, in: Solar Variability: From Core to Outer Frontiers, edited by: Wilson, A., Proc. 10th European Solar Physics Meeting., Prague, Czech Republic, ESA SP-506, 2, 681-684, 2002.

Lin, Y.: Magnetic Field Topology Inferred from Studies of Fine Threads in Solar Filaments, PhD Thesis, Institute of Theoretical Astrophysics, University of Oslo, 2004.

Lin, Y., Engvold, O., and Wiik, J. E.: Counterstreaming in a Large Polar Crown Filament, Sol. Phys., 216, 109-120, 2003.

Lin, Y., Engvold, O., Rouppe van der Voort, L., Wiik, J. E., and Berger, T. E.: Thin Threads of Solar Filaments, Sol. Phys., 226, 239-254, 2005.

Lin, Y., Martin, S. F., Engvold, O., Rouppe van der Voort, L. H. M., and van Noort, M.: Dynamics of an Active Region Filament, Fibrils and Surges in High Resolution, 36th COSPAR Scientific Assembly, Beijing, China, \#3193, in press, 2008.

Litvinenko, Y.: Photospheric Magnetic Reconnection and Canceling Magnetic Features on the Sun, Astrophys. J., 515, 435-440, 1999.

Litvinenko, Y. and Martin, S. F.: Magnetic Reconnection as the cause of a Photospheric Canceling Feature and Mass Flows in a Filament, Sol. Phys., 190, 45-58, 1999.

Litvinenko, Y., Chae, J., and Park, S.-Y.: Flux Pile-up Magnetic Reconnection in the Solar Photosphere, Astrophys. J., 662, 13021308, 2007.

Litvinenko, Y. and Wheatland, M. S.: A Simple Dynamical Model for Filament Formation in the Solar Corona, Astrophys. J., 630, 587-595, 2005.

Martens, P. C. H. and Zwaan, C.: Origin and Evolution of FilamentProminence Systems Astrophys. J., 558, 872-887, 2001. 
Martin, S. F.: Lecture Notes in Physics, IAU Colloquium at Hvar, Yugoslavia, "Dynamics of Solar Prominences", edited by: Rudzjak, V. and Tandberg-Hanssen, E., Springer-Verlag, p. 1, 1990.

Martin, S. F.: Conditions for the Formation and Maintenance of Filaments (Invited Review), Sol. Phys., 182, 107-137, 1998.

Martin, S. F., Bilimoria, R., and Tracadas, P. W.: Magnetic Field Configurations Basic to Filament Channels and Filaments, in: Solar Surface Magnetism, edited by: Rutten, R. J. and Schrijver, C. J., Kluwer Academic Publishers, Dordrecht, p. 303, 1994.

Martin, S. F., Meyer, K., and Gurman, J.: An Experiment in Forecasting Erupting Prominences Using EIT He II (304 ̊̊) Images, presentation at SOHO 17 Workshop, Sicily, 2006.

Nagashima, K., Isobe, H., Yokoyama, T., Ishii, T. T., Okamoto, T. J., and Shibata, K.: Triggering Mechanism for the Filament Eruption on 2005 September 13 in NOAA Active Region 10808, Astrophys. J., 668, 533-545, 2007.

Okamoto, T. J., Nakai, H., Keyama, A., Narukage, N., UeNo, S., Kitai, R., Kurokawa, H., and Shibata, K.: Filament Oscillations and Moreton Waves Associated with EIT Waves, Astrophys. J., 608, 1124, 2004.

Rompolt, B. and Bogdan, T.: On the Formation of Active Region Prominences (H $\alpha$ Filaments), in: Coronal and Prominence Plasmas, edited by: Poland, A. I., NASA Conf. Publ., 2442, 81, 1986.

Rust, D. M.: An active role for magnetic fields in solar flares, Solar Phys., 47, 21-40, 1976.
Smith, S. F.: The Formation, Structure and Changes in Filaments in Active Regions, in: Structure and Development of Solar Active Regions, edited by: Kiepenheuer, K. O., D. Reidel Pub. Co., p. 267-279, 1968.

Svestka, Z., Jackson, B. V., and Machado, M. E. (Eds.): Eruptive Solar Flares, Publisher, Springer-Verlag, New York, p. 33, 1992.

van Ballegooijen, A. A. and Martens, P. C. H.: Formation and Eruption of Solar Prominences, Astrophys. J., 343, 971, 1989.

Wang, Y.-M. and Sheeley Jr., N. R.: Filament Eruptions near Emerging Biples, Astrophys. J., 510, L157-L160, 1999.

Wang, Y.-M. and Muglach, K.: On the Formation of Filament Channels, Astrophys. J., 666, 1284-1295, 2007.

Wood, P. and Martens, P.: Measurements of Flux Cancellation During Filament Formation, Sol. Phys., 218, 123-135, 2003.

$\mathrm{Xu}, \mathrm{X}$. Y., Fang, C., and Chen, P. F.: Statistical study on filament eruption caused by emerging flux, Acta Astronomica Sinica, 48, 181-189, 2007.

Zirker, J. B., Engvold, O., and Martin, S. F.: Counter-streaming Gas Flows in solar Prominences as Evidence for Vertical Magnetic Fields, Nature, 396, 440-441, 1998.

Zwaan, C.: Atmospheric Fine Structure as a Probe for the Solar Interior, in: High Resolution in Solar Physics; Proceedings of the Specialized Session, Toulouse, France, 17-21 September 1984, Springer-Verlag, 263-275, 1985. 